\title{
The sensitivity of a polarimetric sensor embedded in unidirectional and cross-ply composite
}

\section{laminates}

\author{
H. Wang ${ }^{1}$, S.L. Ogin $^{1}$, A.M.Thorne ${ }^{1}$, G.T. Reed ${ }^{2}$ and M Ussorio ${ }^{1}$ \\ ${ }^{1}$ School of Engineering \\ ${ }^{2}$ School of Electronics and Physical Sciences \\ University of Surrey, Guildford, Surrey GU2 5XH, UK
}

\begin{abstract}
Matrix cracking in composite laminates is the first macroscopic damage mode to be readily detected. Polarimetric sensors embedded in composite laminates can detect the development of this damage and they have an advantage over other sensors of being able to sense damage over long gauge lengths (potentially, many metres). In this paper, the sensitivity of a polarimetric sensor manufactured from Hi-Bi PANDA fibre has been measured experimentally and a phase-strain model available in the literature has been used to determine the characteristic parameters of the sensor. The sensitivity of such sensors embedded in unidirectional composites is shown to be in good agreement with theoretical predictions, allowing for material non-uniformity. In the case of cross-ply laminates, which are transversely anisotropic, it is shown that sensor sensitivity is dependent on the relationship of the sensor axes to the composite axes, as well as on the degree of sensor twist. Maximum sensitivity is obtained for a combination of low twist angle and congruence between the sensor optical axes and the composite axes. Twist angles of greater than $90^{\circ}$ give rise to sensitivities, which, although lower, are reasonably constant and approximately the same as the sensitivity of the sensor in a unidirectional composite.
\end{abstract}

\section{Introduction}

The use of fibre optic sensors for monitoring the behaviour of composite structures has gained increasing attention in recent years [e.g. 1-3]. Of the various optical fibre sensors that are available, the conventional optical fibre Bragg grating is now the most widely used, being employed for 
various functions, including point strain measurements and damage detection [e.g. 4,5]. One disadvantage of fibre Bragg gratings for damage detection is their small gauge length. Recent work has shown that a polarimetric sensor embedded in the $0^{\circ}$ ply of a cross-ply composite laminate near the $0 / 90$ interface can detect transverse ply crack formation and, using FFT techniques the stepchange signature of crack development can be distinguished from the background noise so that cracks can be detected in real time [6,7]. An example is shown in figure 1. Figure 1(a) shows the load and strain signal for a $(0 / 90)_{\text {s }}$ cross-ply composite coupon subjected to a uniformly increasing load over a 10 second time period. When a crack forms in the $90^{\circ} \mathrm{ply}$, both the load and the strain (measured using a long gauge length extensometer) respond to the sudden increase in the length of the coupon that occurs due to the local reduction in Young's modulus of the specimen and the relaxation of the thermal residual stress. Figure 1(b) shows the change in the output signal of the polarimetric sensor together with the strain changes recorded by an extensometer. There is a stepchange in the optical output of the sensor when a crack forms, and this step-change only occurs for cracks that form within the gauge length of the polarimetric sensor. Figure 1(c) shows the FFT band pass filtered optical signal, demonstrating that the formation of the crack can be readily detected by such a system (further details can be found in [6]). Although the strain sensitivity of the polarimetric sensor is inferior to other sensors (such as the fibre Bragg grating sensor), the advantage of the polarimetric sensor is that the sensor gauge length (i.e. the distance between the two $45^{\circ}$ splices which define the sensor gauge length) can be very large (metres). Hence, the polarimetric sensor may be useful if damage detection is required in large composite structures, although it should be pointed out that the location of the damage cannot be determined. In this paper, the sensitivity of such a sensor based on PANDA fibre embedded in unidirectional and cross-ply GFRP laminates is determined and the consequences of sensor twist within the composite are investigated.

\section{Determination of the strain-optical parameters of the polarimetric sensor.}

The model proposed by Sirkis $[8,9]$ has been used to determine the parameters that relate the phase change of the polarimetric sensor to the applied strains. According to this model, the relative phase change between the two polarisation modes of the polarimetric sensor, $\Delta \varphi$, is given by:

$$
\Delta \varphi=\frac{2 \pi}{\lambda} \int_{L}\left[K_{1} \varepsilon_{1}+K_{2} \varepsilon_{2}+K_{3} \varepsilon_{3}\right] d L
$$


where $\Delta \varphi$ is the relative phase change caused by the three-dimensional strain, $\lambda$ is the wavelength of light in vacuum, $\varepsilon_{1}, \varepsilon_{2}$ and $\varepsilon_{3}$ are the three normal strains (where the 1-axis is parallel to the length of the sensor), and $\mathrm{L}$ is the gauge length of the sensor. $K_{1}, K_{2}$ and $K_{3}$ are dimensionless parameters to be determined which govern the contribution of each component of the normal strains to $\Delta \varphi$. These $K$ values are characteristic parameters of the optical fibre and are determined by the interaction between the optical fibre core, the Stress Applying Parts (SAPs) and the cladding, due to the differing thermal expansion coefficients of these regions. Previous work by Sirkis, Lo and colleagues $[9,10]$ determined the $K$ values for a polarimetric sensor using Bow-tie optical fibres. In the present study, the polarisation-maintaining fibre used was Fujikura PANDA Hi-Bi fibre, for which the $K$ values have not previously been determined (figure 2 shows a cross-section of the PANDA Hi-Bi fibre embedded in a cross-ply composite coupon; the SAPs can be seen either side of the core).

The single mode, polarisation maintaining fibre (Hi-Bi PANDA fibre obtained from Fujikura Europe Ltd), has a diameter without coating of $125 \mu \mathrm{m}$, a core diameter of $3.5 \mu \mathrm{m}$ and SAP diameter of $16.5 \mu \mathrm{m}$. The protective nylon coating and inner silicone coating were removed from the fibre by a coating stripper and a lens tissue soaked with high purity alcohol (to clean the stripped bare fibre). The gauge length of the polarimetric sensor was achieved in the normal way by a rotation of the axes of the fibre through $45^{\circ}$ at each of two splices, relative to the orientation of the lead-in/leadout sections of the optical fibre. Splicing and rotation of the fibres were carried out using a Fujikura fusion splicer (Model: FSM-20PM). The light source launched into the lead-in fibre was linearly polarised light generated from a He-Ne gas laser source with an operational wavelength of $633 \mathrm{~nm}$; the lead-out fibre was connected to a photodetector and amplifier by a standard connector. The output signal from the amplifier was converted to a voltage signal and recorded by a datalogger.

To determine the K-values, tensile and transverse compression loads were applied to the stripped sensing length of the sensor. Tensile tests of free sensors were carried out in a tension rig within which the sensor was fastened; transverse compression tests used two chucks to grip the sensor so that the response of the sensor as a function of rotation (in $15^{\circ}$ increments) could be found.

Following [9], the longitudinal phase sensitivity of a bare fibre, $S_{\text {axial }}$, is defined as: 


$$
S_{\text {axial }}=\frac{1}{L} \frac{\Delta \varphi}{\Delta \varepsilon_{1}}
$$

where $\mathrm{L}$ is the gauge length of the sensor, $\Delta \varphi$ is the change of phase and $\Delta \varepsilon_{1}$ is the longitudinal strain change. Five free sensors were tested with lengths in the range $102 \mathrm{~mm}$ to $120 \mathrm{~mm}$ and the average longitudinal sensitivity of the free sensors was $106 \pm 2 \mathrm{rad} / \mathrm{mm}$, where the uncertainty is the standard deviation. The transverse sensitivity, $\mathrm{S}_{\text {trans }}$, of the sensor under compression is defined as:

$$
S_{\text {trans }}=\frac{1}{L} \frac{\Delta \varphi}{\Delta P}
$$

where $\Delta P$ is the change of load per unit length on the sensor, $\Delta \varphi$ is the phase change induced by $\Delta P$, and $L$ is the length of the sensor under transverse compression. The results showing the change of sensitivity with rotation angle, $\theta$, are plotted in figure 3(a) and the dashed line indicates the trend of the data; the angle $\theta$ is defined in Figure 3(b). The sensitivity as a function of angle in figure 3(a) shows four peaks, which correspond to the loading of the sensor parallel to the optical axes. Peaks labeled $\mathrm{A}$ and $\mathrm{B}$ correspond to angles of $\theta=0^{\circ}$ and $\pi$; peaks labeled $\mathrm{C}$ and $\mathrm{D}$ correspond to angles of $\pi / 2$ and $3 \pi / 2$.

The mechanical properties used for the optical fibre are shown in Table 1 [8]. From figure 3(a), the experimental values for the sensitivity at the peaks for angles of 0 and $\pi$ are $0.47 \mathrm{rad} / \mathrm{mm}$ and for $\pi / 2$ and $3 \pi / 2$ the value is $0.63 \mathrm{rad} / \mathrm{m}$. The method followed to extract the values of $K_{1}, K_{2}$ and $K_{3}$ from such experimental results has been described in detail in [9]. It consists of constructing three simultaneous equations for $K_{1}, K_{2}$ and $K_{3}$, one equation arising from the longitudinal loading of the sensor and two from the transverse loading (at $0 \& \pi$ radians and $\pi / 2 \& 3 \pi / 2$ radians, respectively). For the transverse loading, the strain within the optical fibre is obtained by considering the transverse loading of a cylindrical cylinder. The resulting K-values for the PANDA fibre are compared with the $\mathrm{K}$ values for a polarimetric sensor based on Bow-tie fibre [9] in Table 3. It is clear that the parameters for the PANDA fibre are smaller than those for the Bow-tie fibre, and this is in agreement with previous work [11], where it was found that a polarimetric sensor based on PANDA fibre is less sensitive to longitudinal strain than a sensor based on Bow-tie fibre. Both 
PANDA and Bow-tie fibres are more sensitive to transverse strain than longitudinal strain (i.e. $K_{2}$ and $K_{3}$ are both larger than $K_{1}$ ).

Having determined the K-values for the PANDA, it is now possible to predict the sensitivity of a sensor embedded within a composite material. In the next section, this prediction is compared with experimental results for a sensor embedded in a unidirectionally reinforced glass/epoxy composite. 


\section{Sensitivity of a polarimetric sensor embedded in a unidirectional composite}

The frame-winding method used to incorporate the optical fibres into transparent glass/epoxy composites has been described elsewhere [e.g. 12]. Sensors incorporated into composites are inevitably twisted along their length, and the consequences of this twist for polarimetric sensors are discussed below. Plain rectangular test coupons, about $230 \mathrm{~mm}$ in length and $20 \mathrm{~mm}$ wide, were cut from laminates using a water-lubricated diamond saw with a nominal 600-grade grit finish. Coupons were postcured after cutting at $150^{\circ} \mathrm{C}$ for 3 hours. After post-curing, aluminium alloy end tabs, 20 $\mathrm{mm}$ long and $20 \mathrm{~mm}$ wide, were bonded to the coupon to avoid damage to the surface plies in the testing machine grips during loading. A glue seat for a $50 \mathrm{~mm}$ extensometer was also applied onto the coupon before mechanical testing to ensure that the extensometer knife-edge remained in a fixed position on the coupon's surface during testing. The coupons were tested in load control using an Instron servohydraulic testing machine $(8000)$ at a loading rate of $0.25 \mathrm{kN} / \mathrm{s}$. Load and strain were recorded using a datalogger at a rate which could vary from $100 \mathrm{~Hz}$ to $1.667 \mathrm{kHz}$, with the optimum rate a compromise between a reasonable file size (below $20 \mathrm{MB}$ ) and low noise. A schematic of the test arrangement is shown in figure 4.

The results for the five sensors tested are shown in Table 4, and the average longitudinal sensitivity was found to be $136 \pm 9 \mathrm{rad} / \mathrm{mm}$. A typical example of fringes for loading and unloading a coupon, where the light intensity (arbitrary units) is plotted against strain (\%) is shown in figure 5(a). The figure shows a specimen loaded to a maximum strain of about $0.4 \%$ and then unloaded; the fringes for loading and unloading superimpose. The predicted longitudinal sensitivity of a polarimetric sensor embedded in a unidirectional composite is given by

$$
S_{\text {axial }}=\frac{1}{L} \frac{\Delta \varphi}{\Delta \varepsilon 1}=\frac{2 \pi}{\lambda}\left(K_{1}-v^{\prime} K_{2}-v^{\prime} K_{3}\right)
$$

where $v^{\prime}$ is the effective Poisson's ratio of the embedded sensor, calculated to be 0.169 for the combination of sensor and GFRP composite used here (Tables 1 and 2), based on the expressions provided in [8] for the relationship between the longitudinal and transverse strains for a sensor embedded parallel to the fibres in a unidirectional composite. Note that the effective Poisson's ratio 
of the embedded optical fibre is greater than the Poisson's ratio of the free fibre (0.154), because the Poisson's ratio of the surrounding composite is much higher, $v_{12}=0.326$ (the complete GFRP lamina properties are shown in Table 2). Based on equation (4), the predicted sensitivity for the polarimetric sensor embedded in the unidirectional composite is $170 \mathrm{rad} / \mathrm{mm}$, which is somewhat higher than the experimentally determined value of $136 \pm 9 \mathrm{rad} / \mathrm{mm}$.

A number of issues arise from these results. First, the transverse Poisson's ratio of the host material $\left(v_{12}=0.326\right)$ is larger than that of the embedded optical fibre $\left(v_{f}=0.154\right)$, which means the host material is compressing the optical fibre when the host material is under tension. This will increase the effective Poisson's ratio of the optical fibre and the sensitivity of the sensor (as mentioned above); hence both the predicted and the experimentally measured sensitivities of the embedded polarimetric sensor are larger than the sensitivity of the bare fibre $(106 \pm 2 \mathrm{rad} / \mathrm{mm})$. Second, the experimental sensitivity of the sensor embedded in the unidirectional composite $(136 \mathrm{rad} / \mathrm{mm})$ is about $20 \%$ less than the predicted value $(170 \mathrm{rad} / \mathrm{mm})$. This is likely to be because the theoretical model assumes that the sensor is a cylindrical inclusion embedded in a transversely isotropic host. Consequently, if some part of the surrounding host composite material is less stiff (due, for example, to a resin rich region adjacent to the sensor), then the compressive effect of the surrounding unidirectional composite material (which has a higher Poisson's ratio) will be reduced and the sensitivity of the embedded sensor will be reduced as well. Third, the embedded sensors have a larger standard deviation for the experimentally measured sensitivities $(9 \mathrm{rad} / \mathrm{mm})$ compared to the free sensors ( $2 \mathrm{rad} / \mathrm{mm})$. This difference is due to the irregularity of the fringe width and spacing for the embedded sensors, as shown in figure 5(a). Although the fringes are irregular, they are reproducible for both loading and unloading, suggesting that the embedded sensors are sensitive to variations in local transverse strains due to the irregular packing of the structural glass fibres around the sensor. To test this hypothesis, a sensor still enclosed within its original soft silicone inner coating and nylon outer coating was embedded into a unidirectional coupon. The optical fringes for a sensor with its coatings and are uniform (see figure 5(b)), and the widths of the optical fringes are almost identical. Hence, it can be concluded that with the coatings intact, the relatively soft silicone inner coating and the nylon outer coating have smoothed the irregularity of the strain field in the vicinity of the sensor due to resin rich regions or structural fibre bunching. Unfortunately, the sensor with its inner and outer coating intact is approximately $1 \mathrm{~mm}$ in diameter, which is too large for most applications in composite materials where the ply thickness is typically $0.125 \mathrm{~mm}$. Finally, a 
unidirectional composite is transversely isotropic in the fibre direction. Hence, the sensitivity of a polarimetric sensor embedded within a unidirectional composite is independent of the angle between the optical axes of the sensor and the composite axes. This is not the case for a sensor embedded within a cross-ply laminate, discussed in the following section, since a cross-ply laminate is not transversely isotropic.

\section{Sensitivity of a polarimetric sensor embedded in a cross-ply composite}

When considering the sensitivity of a sensor embedded in a cross-ply laminate, there are two sets of axes to consider, the coupon axes and the optical axes of the sensor, and the definition of the axes used here is shown in figure 6. Figure 6(a) shows the composite axes and figure 6(b) shows a crosssection of the coupon, with the optical axes 2 (joining the SAPs) and 3 (perpendicular to the SAPs) shown. The angle $\theta$ between the optical axes of the sensor and the axes of the coupon is not constant along the sensor length because the embedded optical fibres have an unavoidable twist introduced during fabrication of the laminate. To determine the magnitude of the twist, coupons were cut into sections after testing and optical microscope images were taken at different positions along the coupon (i.e. for different $\mathrm{z}$ co-ordinates as defined in figure 6(a)) in order to determine the angle $\theta$ as a function of distance $\mathrm{z}$. These measurements enable the degree of twist to be defined at any distance $\ell$ along the sensor from one splice (the reference splice). The orientation of the fibre at any point along its length can be expressed in terms of the angle $\theta$ as:

$$
\theta_{\ell}=\theta_{0}+\frac{z}{L_{0}} \theta
$$

where $\theta_{\ell}$ is the orientation of the embedded sensor at distance $z$ within the gauge length of the sensor from the reference splice; $L_{0}$ is the gauge length of the sensor, $\theta_{0}$ is the initial orientation of the sensor at the reference splice $(\mathrm{z}=0)$ and $\theta$ is the overall twist of the sensor measured from the first to the second splice, defined as positive for anti-clockwise rotation. These parameters, which define the initial orientation of the sensor at the first splice and the degree of twist, were measured in addition to the sensitivity of the sensors.

Figure 7 shows a typical result for the measurement of the twist of the sensor. Figure 7(a) shows optical micrographs of four sections at various positions along a coupon $(z=10 \mathrm{~mm}, 34 \mathrm{~mm}, 64 \mathrm{~mm}$ 
and $86 \mathrm{~mm}$ ) and figure 7(b) shows the linearity of the relationship between the twist angle, $\theta$, and distance from the first splice. This linearity was a feature of each embedded sensor, although the twist rate varied between specimens.

Table 5 shows the initial orientation, $\theta_{0}$, of the sensor axes at the first splice, the overall twist between the splices, the sensor length and the measured sensitivity for all the sensors tested. The results in Table 5 show no correlation between sensor sensitivity and either the initial fibre orientation or the sensor length. However, when the overall twist angle of the sensor, is plotted against the sensitivity, as in figure 8, a clear pattern emerges. For overall twist angles above about $90^{\circ}$, the sensor sensitivity is approximately constant at about $145 \pm 15 \mathrm{rad} / \mathrm{mm}$. This value is very similar to the sensitivity found for the sensors embedded in the unidirectional composites i.e. $136 \pm 9$ $\mathrm{rad} / \mathrm{mm}$ (see Section 3). Specimens 1, 3 and 6, which have much higher values of sensor sensitivity, have small overall twist angles and initial orientation angles at the first splice. Hence, the strain field experienced by these sensors is dominated by the congruence between the optical axes and the composite axes. As figure 3(a) shows, the transverse sensitivity of the polarimetric sensor is strongly dependent on the direction of loading, showing peaks when loaded either parallel to the line joining the SAPs or when loaded perpendicular to this direction. Hence, the sensitivity of the sensors in specimens 1,3 and 6 would be expected to be high. For the other sensors, the larger twist angles suggest that the sensitivity will be related to the response of the sensor averaged over at least one peak and trough of the sensitivity response (figure 3(a)). Indeed, one way to picture the effect of these large overall twist angles on the sensor response is to imagine that the sensor remains straight but the surrounding 0/90 composite rotates in a helical fashion along the sensor length. For a sensor embedded in the $0^{0}$ ply close to the $0 / 90$ interface, this would be equivalent to embedding a sensor without twist within a transversely isotropic composite consisting of fibres parallel to the sensor direction (the $0^{0}$ fibres), surrounded by helically-rotated $90^{\circ}$ fibres. Viewed in this way, it is not surprising that the sensitivity of sensors with twist angles of $\pi / 2$ or greater is approximately the same as the sensitivity of sensors embedded in unidirectional composites. 


\section{Conclusions}

The dimensionless $K$ parameters, which govern the relationship between the normal strains and the sensitivity of a polarimetric sensor, have been measured for a PANDA fibre and found to be lower than for a Bow-tie fibre. When embedded in a unidirectional composite, the interference fringes from a bare polarimetric sensor are irregular in amplitude and period, but reproducible for loading and unloading. This irregularity is probably due to the non-uniformity of the strain field in the vicinity of the optical fibre due to irregular packing of the adjacent structural fibres. The experimental measurement of sensor sensitivity is about $20 \%$ lower than predicted, which is again due to the non-uniform strain field in the vicinity of the optical fibre, especially the effect of local reductions in the value of the Poisson's ratio due to resin-rich regions. The response of polarimetric sensors embedded within unidirectional composites is independent of sensor orientation or sensor twist because of the transversely isotropic nature of the host material. On the other hand, the sensitivity of a polarimetric sensor embedded in the $0^{0}$ ply of a cross-ply composite is dependent on the total twist of the sensor over the sensor gauge length. Large twist angles (i.e. greater than $90^{\circ}$ ) produce sensitivities that are very similar to the results for sensors embedded in unidirectional composites. Hence, if constant values of the strain sensitivity are required, steps should be taken to ensure that the sensors are twisted to angles of $90^{\circ}$ or more before being embedded in a composite structure.

\section{Acknowledgements}

The authors are grateful to the Overseas Research Students Award Scheme (ORSAS) for the provision of funding for $\mathrm{H}$ Wang, and to $\mathrm{Mr} \mathrm{R}$ Whattingham for assistance in the manufacture of composite specimens. 


\section{References}

(1) Culshaw B, Michie C, Gardiner P and McGown A (1996), "Smart structures and applications in civil engineering", Proc of the IEEE, 84, pp.78-86.

(2) Kersey A, Davis M A., Patrick H J, LeBlanc M, Koo K P, Askins C G, Putnam M A., Friebele J , (1997), "Fiber Grating Sensors." Journal of Lightwave Technology,15, pp.1442-1463.

(3) Botsis J, Humbert L, Colpo F and Giaccari (2005), "Embedded fiber Bragg gratings sensor for internal measurements in polymeric materials." Optics and Lasers Engineering, 43, pp. 491-510

(4) Okabe Y, Yashiro S, Kosaka T and Takeda N, (2000). "Detection of transverse cracks in CFRP composites using embedded fiber Bragg grating sensors." Smart Materials and Structures, 9, pp. 832-838

(5) Gebremichael Y M, Li W, Boyle W J O, Meggitt B T, Grattan K T V, McKinley B, Fernando G F, Kister G, Winter D, Canning L and Luke S (2005), "Integration and assessment of fibre Bragg grating sensors in an all-fibre reinforced polymer composite road bridge.", Sensors and Actuators, A118, pp.78-85

(6) Wang H., Ogin S.L., Thorne A.M and Reed G.T. (2002), "Use of a polarimetric sensor for damage detection in cross-ply composite laminate," in Proceedings of ECCM-10, The Tenth European Conference on Composite Materials, Brugge, Belgium

(7) Wang H., Ogin S.L., Thorne A.M and Reed G.T (2006), "Interaction between optical fibre sensors and matrix cracks in crossply GFRP laminates. Part 2: Crack detection”, Composites Science Technology, 66, pp. $2367-2378$

(8) Sirkis J S (1993), "Unified approach to phase-strain-temperature models for smart structure interferometric optical fibre sensors: Part 1, Development; Part 2, Applications", Optical Engineering, 324, pp. $752-773$.

(9) Sirkis J S, Lo Y L and Nielsen P L (1994), "Phase-strain model for polarimetric strain sensors based on fictitious residual strains". Journal of Intelligent Materials Systems and Structures, 5, pp.494-500.

(10) Lo Y I, Sirkis J S and Ritchie K T (1995), "A study of the optomechanical response of a diametrically loaded high-birefringent optical fibre", Smart Materials and Structures, 4, pp. 327-333.

(11) Tsai K., Kim K, and Morse T (1991) "General solutions for stress-induced polarisation in optical fibres", Journal of Lightwave Technology, 9, pp. 7-17

(12) Barton E N, Ogin S L, Thorne A M and Reed G T (2001) "Interaction between optical fibre sensors and matrix cracks in cross-ply GRP laminates-part 1: passive optical fibres", Composites Science and Technology, 13(61), pp. 1863-1869 


\section{Tables}

\begin{tabular}{|c|c|}
\hline Property & Value \\
\hline Young's modulus, E $(\mathrm{GPa})$ & 73.3 \\
\hline Poisson's ratio, $v$ & 0.154 \\
\hline Diameter of the optical fibre, $\mathrm{d}(\mu \mathrm{m})$ & 125 \\
\hline
\end{tabular}

Table 1. Optical fibre properties [8]

\begin{tabular}{|c|c|}
\hline Property & Value \\
\hline Longitudinal Young's modulus, $E_{l}(\mathrm{GPa})$ & 39 \\
\hline Transverse Young's modulus, $E_{2}(\mathrm{GPa})$ & 11 \\
\hline Transverse Young's modulus, $E_{3}(\mathrm{GPa})$ & 4.6 \\
\hline Shear modulus, $G_{23}(\mathrm{GPa})$ & 5 \\
\hline Shear modulus, $G_{21}(\mathrm{GPa})$ & 5 \\
\hline Shear modulus, $G_{31}(\mathrm{GPa})$ & 0.303 \\
\hline Poison's ratio, $v_{23}$ & 0.326 \\
\hline Poison's ratio, $v_{12}=v_{13}=v_{21} \cdot E_{1} / E_{2}$ & 0.092 \\
\hline Poison's ratio, $v_{21}$ & 1 \\
\hline$b$, thickness of $0^{\circ} \mathrm{ply}, \mathrm{mm}$ & 0.5 \\
\hline$d$, half thickness of $90^{\circ} \mathrm{ply}, \mathrm{mm}$ & \\
\hline
\end{tabular}

Table 2 Composite properties

\begin{tabular}{|c|c|c|c|}
\hline Fibre & $\mathrm{K}_{1}$ & $\mathrm{~K}_{2}$ & $\mathrm{~K}_{3}$ \\
\hline PANDA & -0.055 & -0.204 & -0.225 \\
\hline Bow-tie & -0.197 & -0.714 & -0.618 \\
\hline
\end{tabular}

Table 3. Measured K-values for the polarimetric sensors based on PANDA fibre compared with similar values for Bow-tie fibres [9]. 


\begin{tabular}{|c|c|c|}
\hline Specimen No. & Sensor gauge length $(\mathrm{mm})$ & $\begin{array}{c}\text { Experimental sensitivity, } \\
\mathrm{rad} / \mathrm{mm}\end{array}$ \\
\hline 1 & 97 & 137 \\
\hline 2 & 97 & 121 \\
\hline 3 & 98 & 144 \\
\hline 4 & 86 & 143 \\
\hline 5 & 89 & 134 \\
\hline
\end{tabular}

Table 4. Sensor sensitivities for sensors embedded in unidirectional glass/epoxy.

\begin{tabular}{|c|c|c|c|c|}
\hline $\begin{array}{c}\text { Specimen } \\
\text { No. }\end{array}$ & $\begin{array}{c}\text { Initial } \\
\text { orientation } \\
\text { angle, }\end{array}$ & $\begin{array}{c}\text { Overall twist }^{\text {angle, }}{ }^{\circ} \\
10 \pm 3\end{array}$ & $\begin{array}{c}\text { Sensor } \\
\text { length } \\
(\mathrm{mm})\end{array}$ & $\begin{array}{c}\text { Experimental } \\
\text { sensitivity, } \\
\text { rad/mm }\end{array}$ \\
\hline 1 & $-116 \pm 3$ & $162 \pm 5$ & 97 & $254 \pm 5$ \\
\hline 2 & $-19 \pm 3$ & $52 \pm 5$ & 91 & $237 \pm 5$ \\
\hline 3 & $-19 \pm 3$ & $228 \pm 5$ & 99 & $160 \pm 5$ \\
\hline 4 & $24 \pm 3$ & $144 \pm 5$ & 95 & $162 \pm 5$ \\
\hline 5 & $-29 \pm 3$ & $64 \pm 5$ & 96 & $207 \pm 5$ \\
\hline 6 & $30 \pm 3$ & $252 \pm 5$ & 98 & $136 \pm 5$ \\
\hline 7 & $-63 \pm 3$ & $124 \pm 5$ & 101 & $118 \pm 5$ \\
\hline 8 & $-77 \pm 3$ & $80 \pm 5$ & 88 & $149 \pm 5$ \\
\hline 9 & $85 \pm 3$ & $168 \pm 5$ & 91 & $140 \pm 5$ \\
\hline 10 & & & & \\
\hline
\end{tabular}

Table 5. Sensor twist parameters and sensitivities for sensors embedded in cross-ply coupons. 


\section{Figure captions}

Figure 1 . Transverse ply crack detection by a polarimetric sensor embedded in the $0^{0}$ ply of a crossply laminate (a) load and strain signals during crack formation; (b) optical output from the sensor and strain signal; (c) FFT band pass filtered optical signal.

Figure 2 A cross-section image of a PANDA fibre embedded in the $0^{0}$ ply of a crossply composite coupon. The optical fibre diameter is $125 \mu \mathrm{m}$.

Figure 3. (a) Sensitivity of the bare sensor under transverse compression as a function of angle $\theta$; (b) definition of the angle $\theta$, which is the angle between the 2-axis and the loading direction (P).

Figure 4. Schematic of the experimental arrangement for measuring the sensitivity of polarimetric sensors embedded in unidirectional and cross-ply composite coupons.

Figure 5. (a) Optical fringes from a bare polarimetric sensor embedded in a unidirectional coupons; (b) optical fringes from an embedded polarimetric sensor with inner and outer coating intact.

Figure 6. (a) Schematic diagram showing the cross-ply laminate axes; (b) the axes of the optical sensor, 2 and 3 , and their relation to the coupon axes, $\mathrm{x}, \mathrm{y}$.

Figure 7. (a) Microsgraphs of sensor cross-section showing twist of the sensor at distances of $z=10$ $\mathrm{mm}, 34 \mathrm{~mm}, 64 \mathrm{~mm}$ and $86 \mathrm{~mm}$ from the first splice (clockwise from top left); (b) measured twist angle as a function of distance, $\mathrm{z}$, from the first splice.

Figure 8. Sensitivity of sensors embedded in a cross-ply laminate as a function of the overall angle of twist of the sensor between the two splices.

\section{Figures}




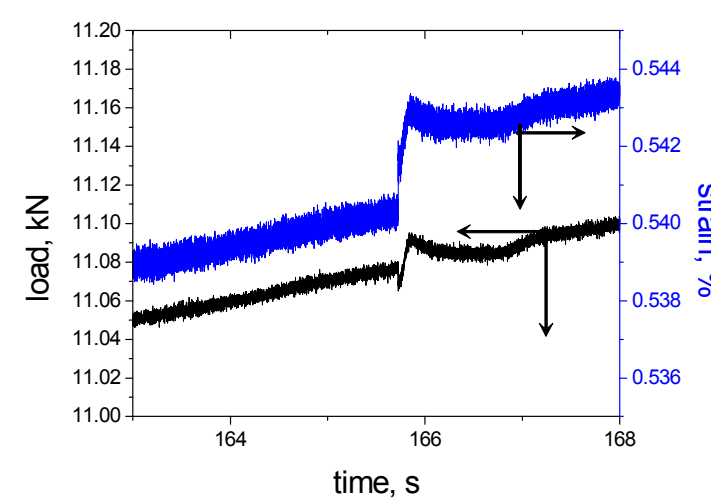

(a)

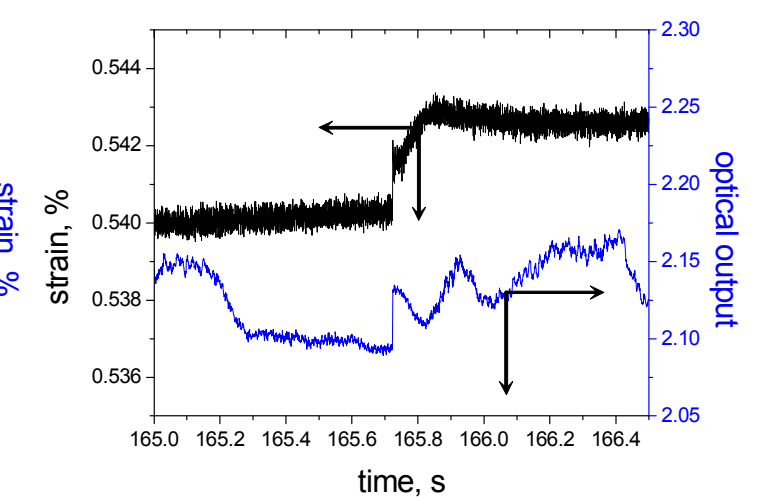

(b)

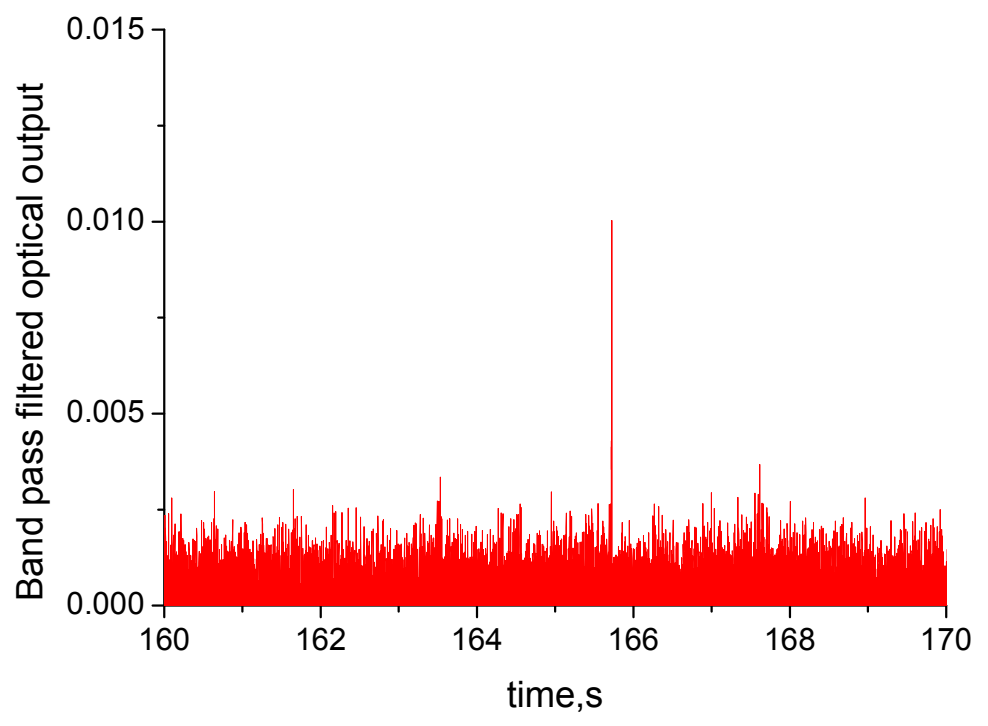

(c)

Figure 1 


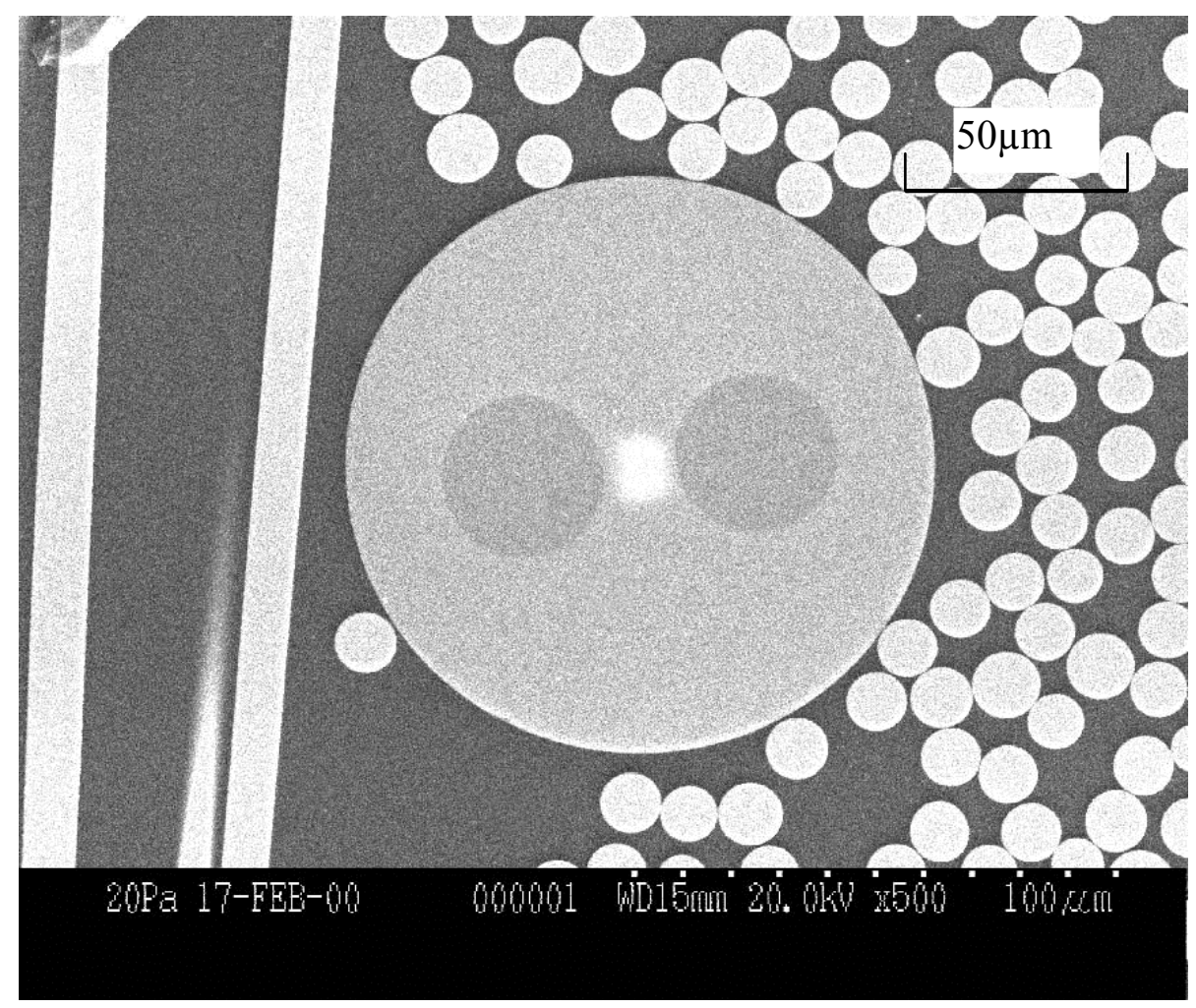

Figure 2. 


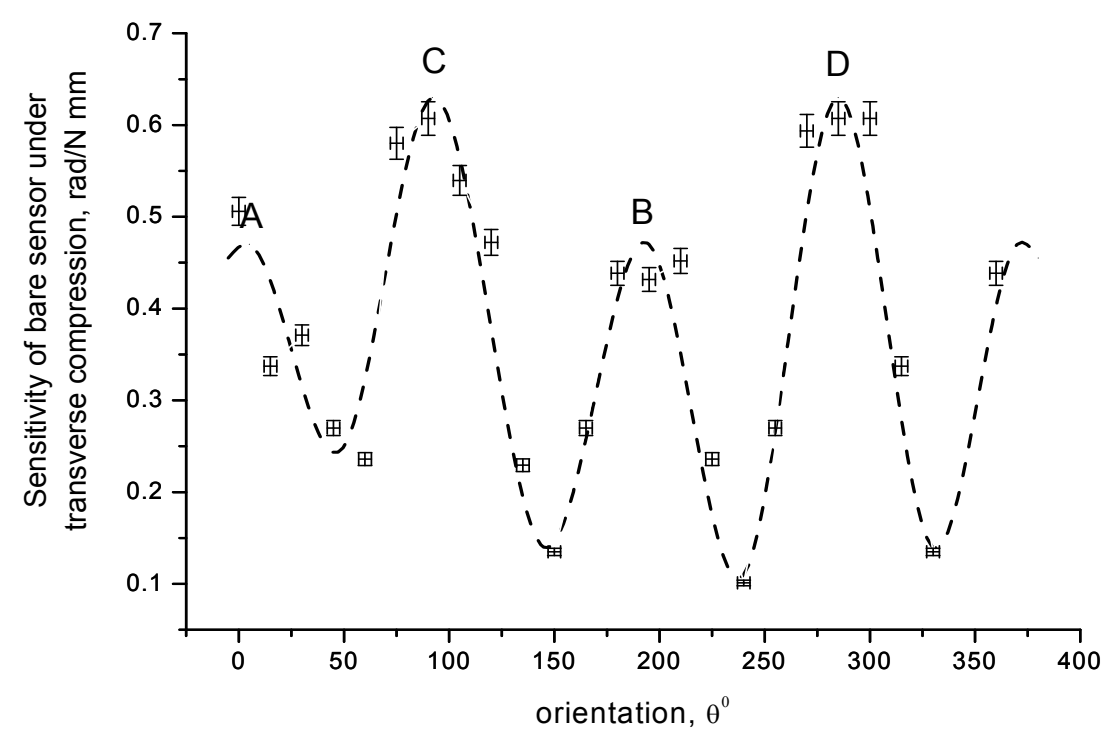

Figure 3(a)

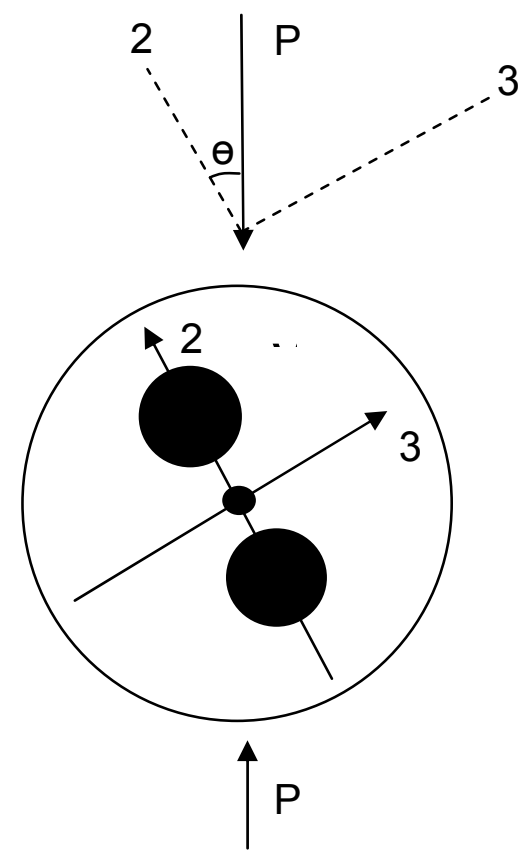

Figure 3(b) 


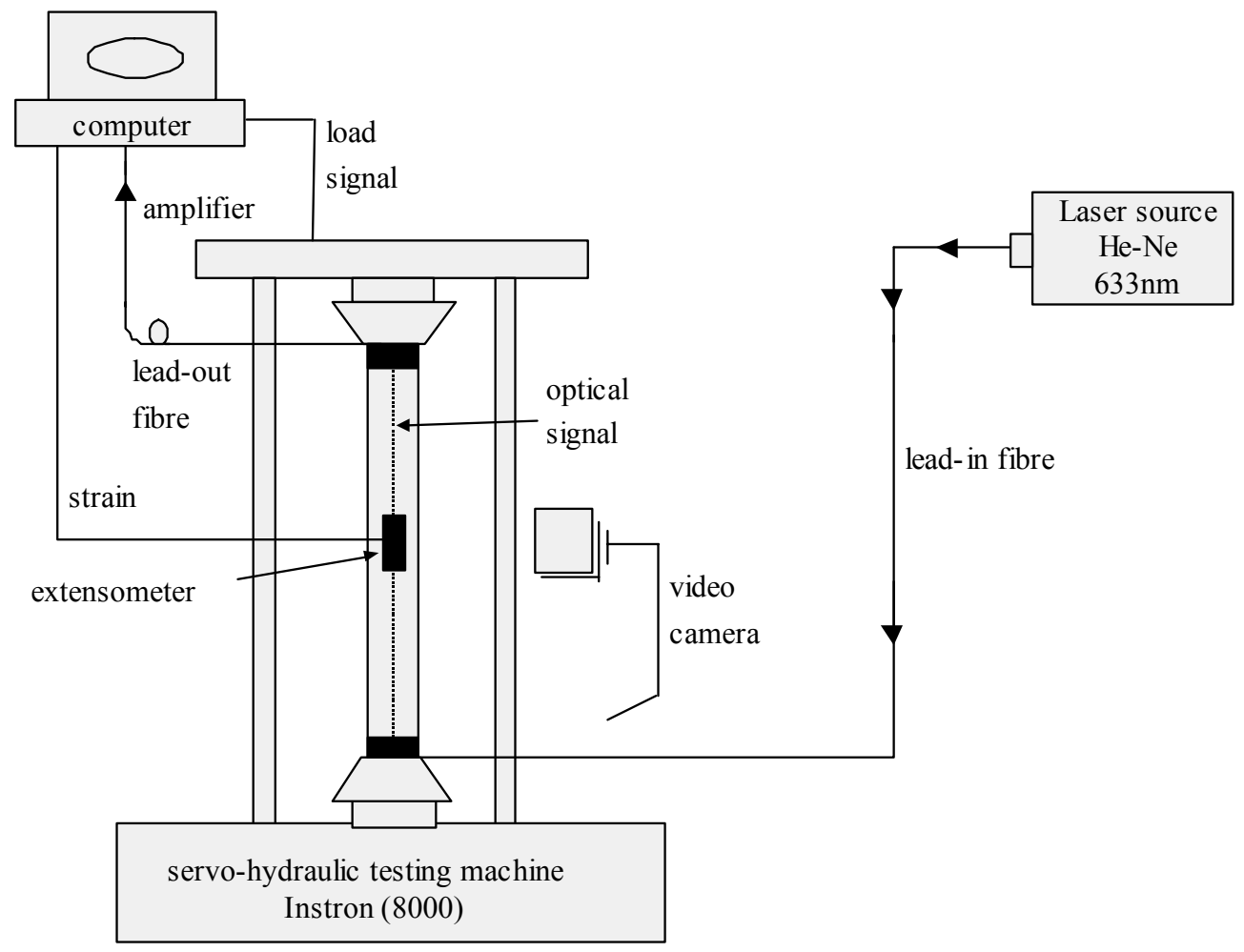

Figure 4. 


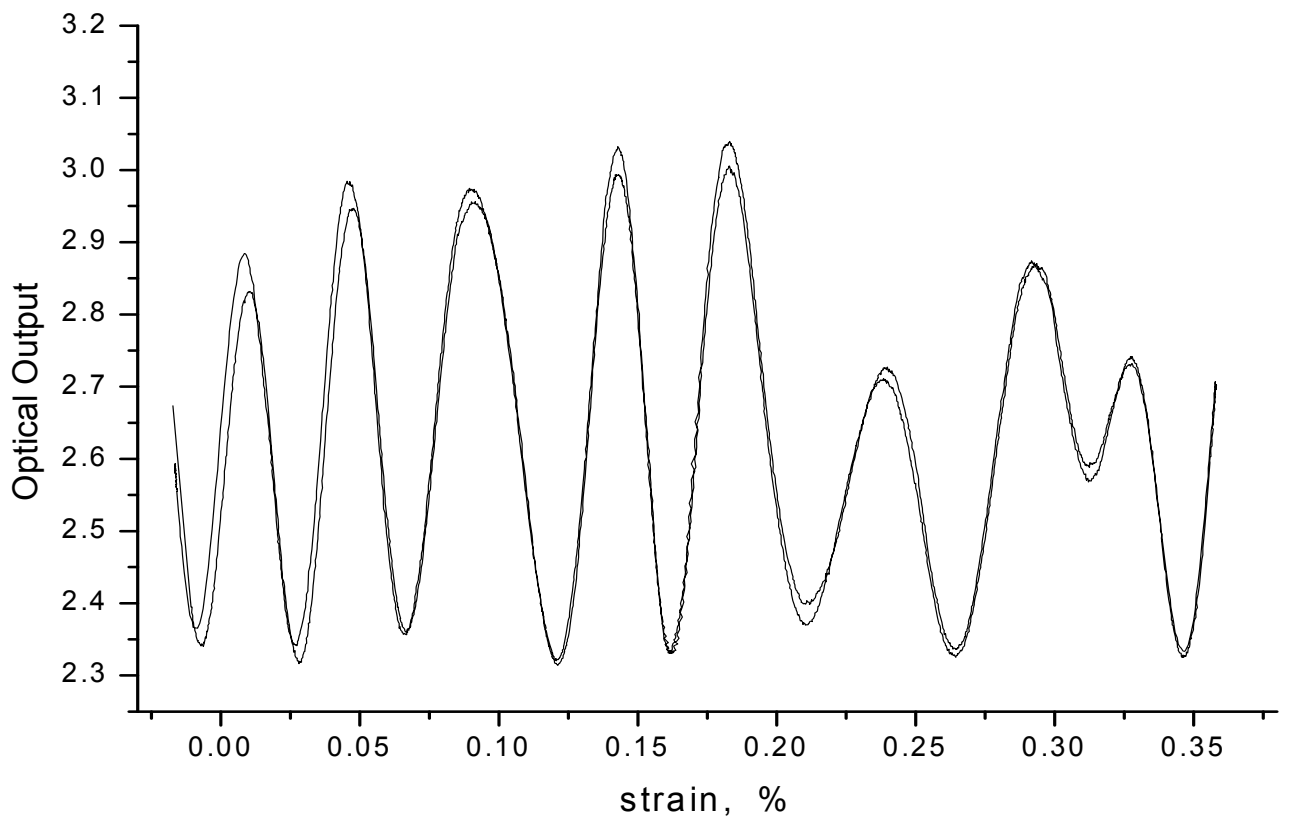

Figure 5(a)

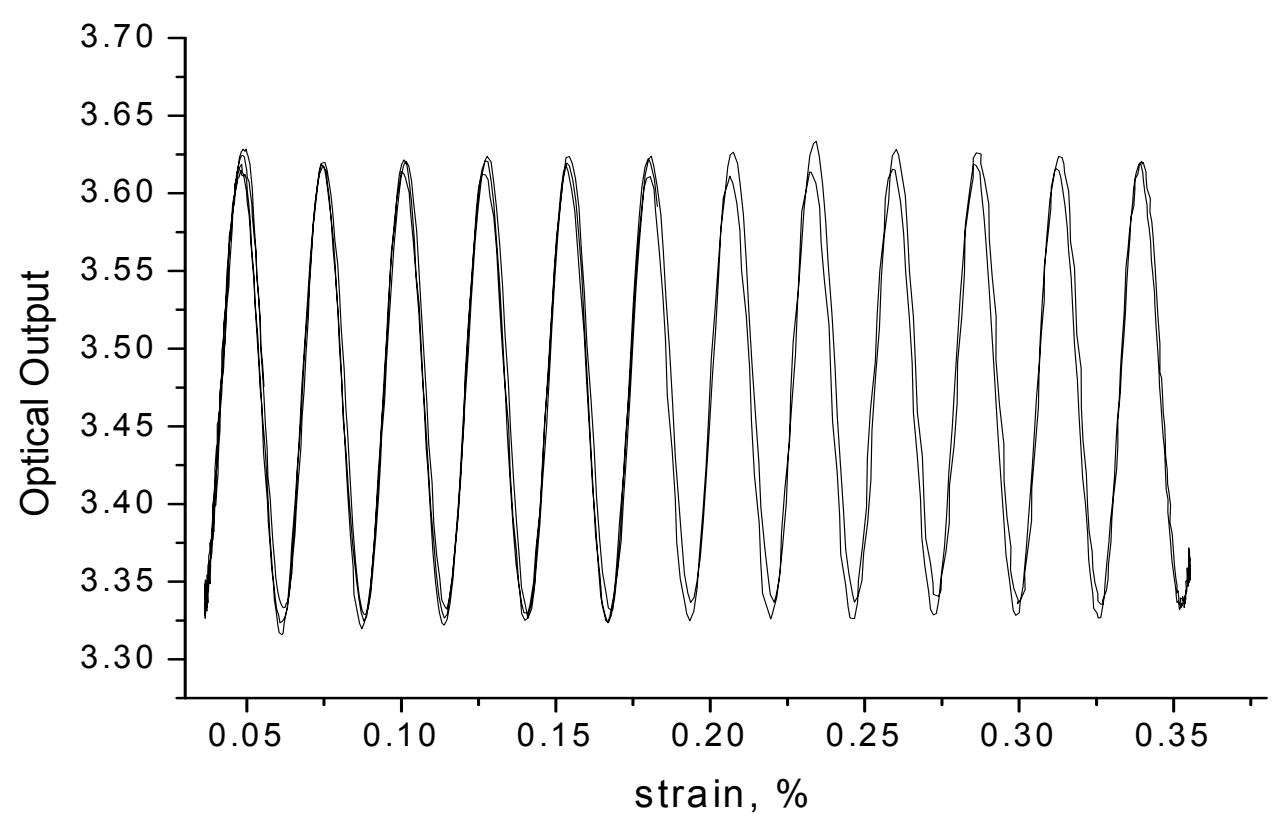

Figure 5(b). 


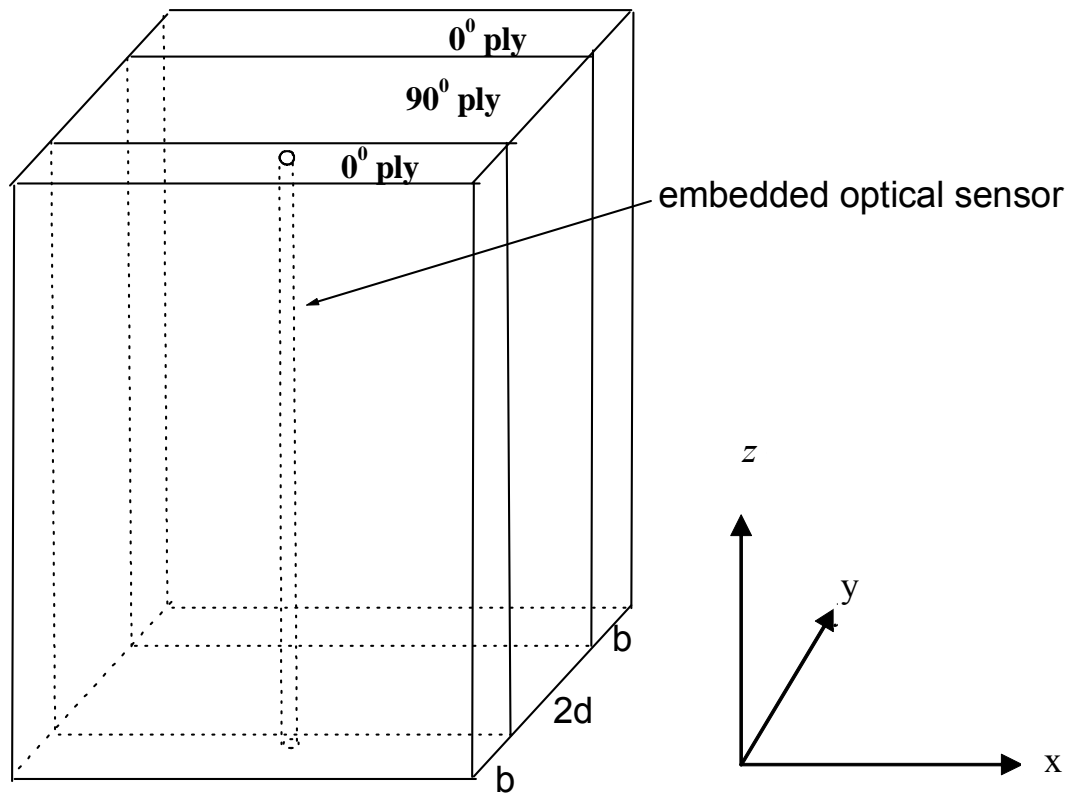

(a)

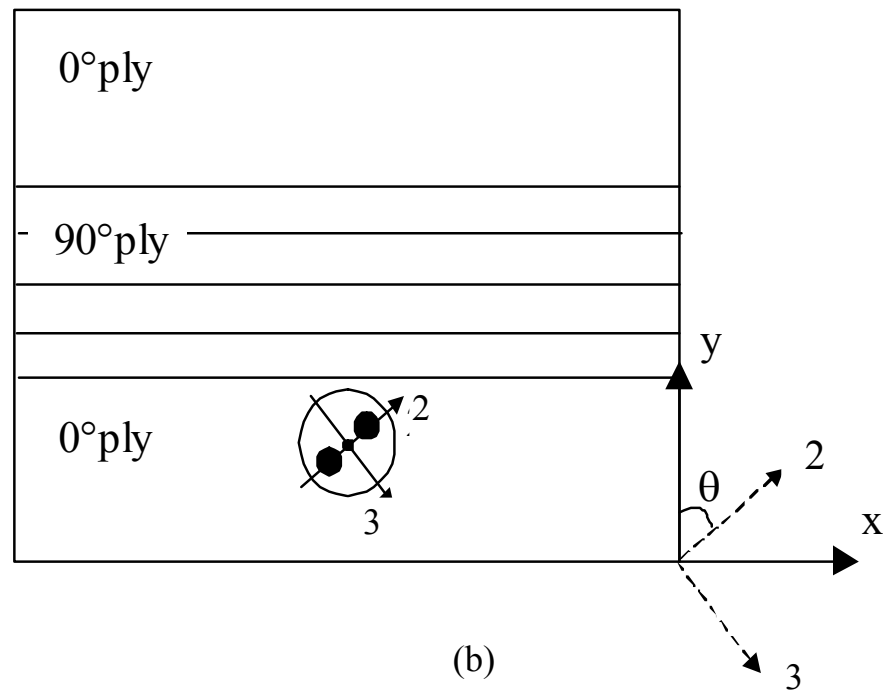

Figure 6. 

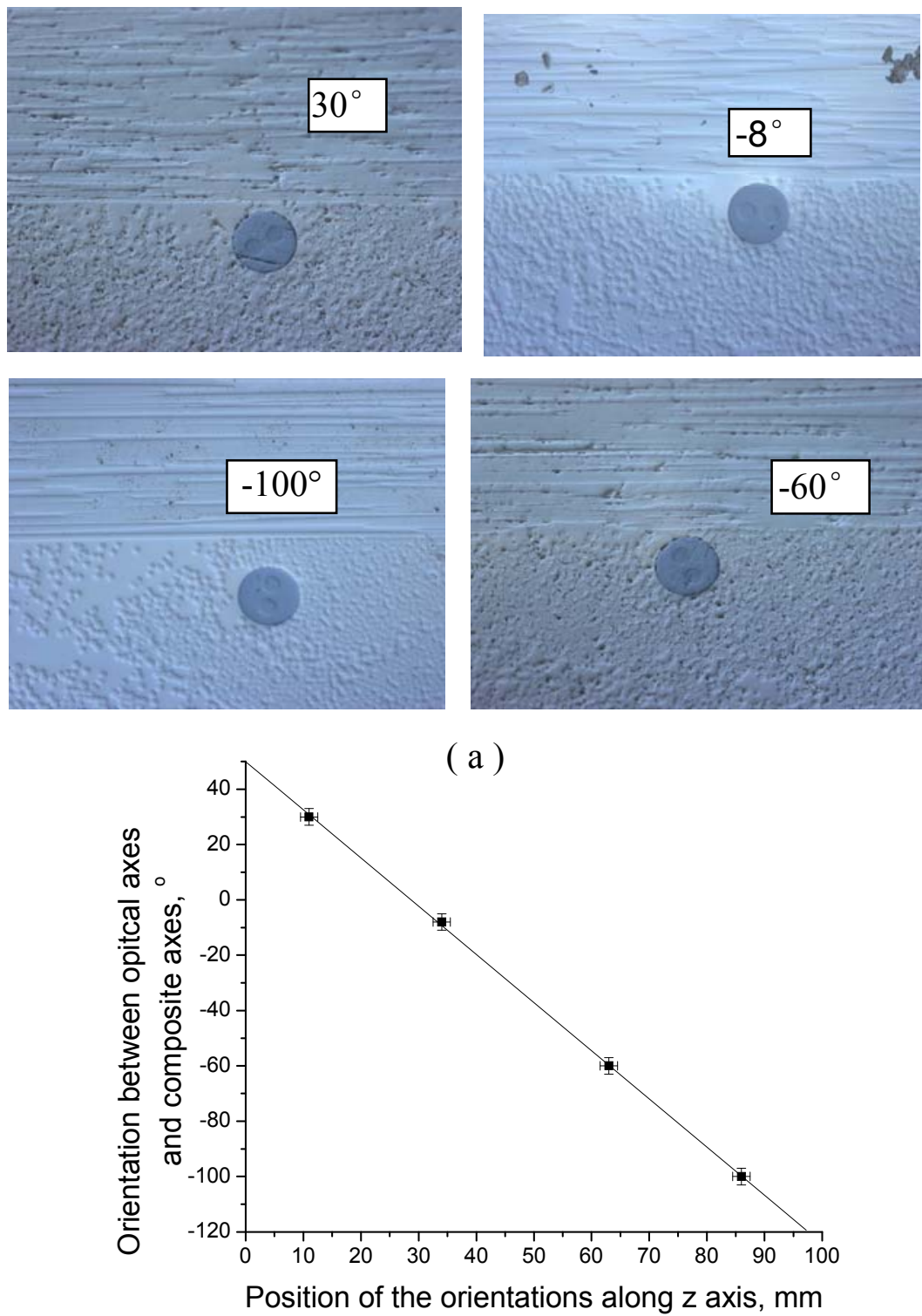

(b)

Figure 7 


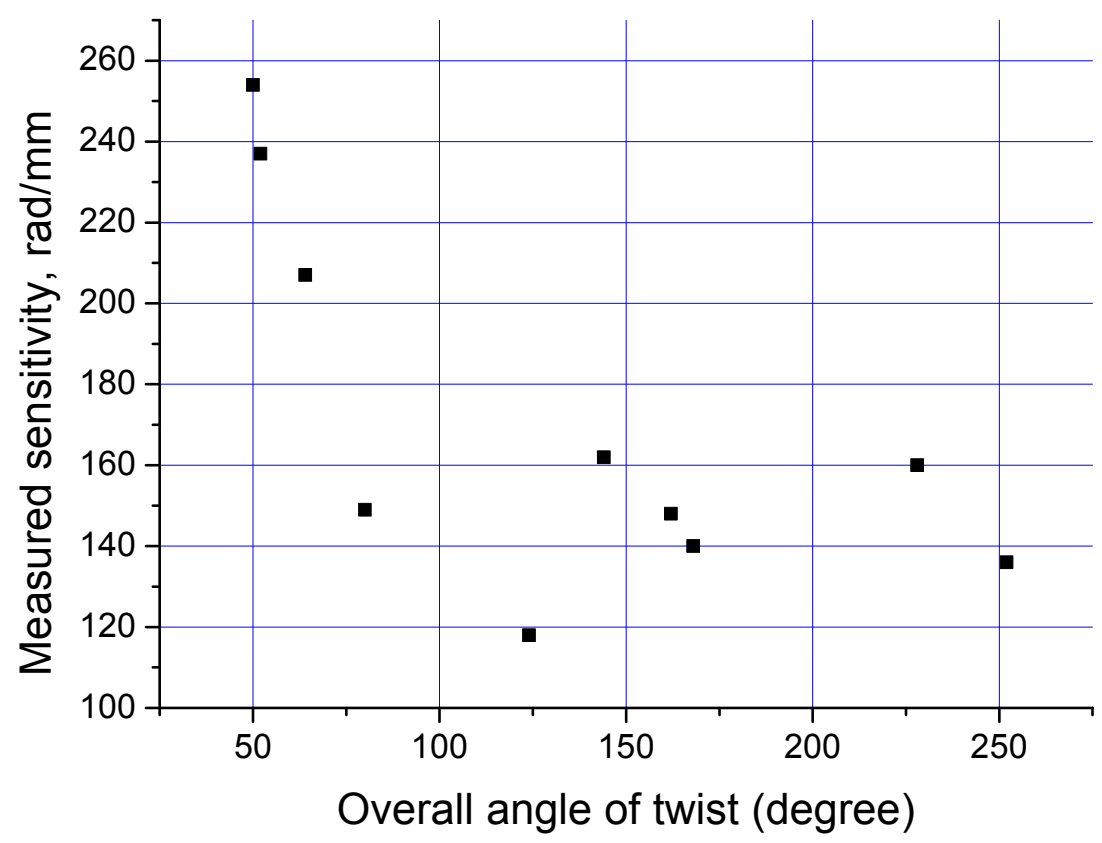

Figure 8 . 